\title{
Mixed adenoneuroendocrine carcinoma of proximal stomach; a rare but sinister pathology Deepshikha Gaire ${ }^{1}$, Daisy Maharjan ${ }^{1}$, Nisha Sharma ${ }^{1}$ \\ ${ }^{1}$ Department of Pathology, Tribhuvan University Teaching hospital, Kathmandu, Nepal
}

\section{Keywords:}

Gastric carcinoma; Immunohistochemistry; MANEC;

\begin{abstract}
Mixed adeno-neuroendocrine carcinoma is a rare tumor of the gastrointestinal tract comprising of both epithelial and neuroendocrine components, each representing at least $30 \%$ of the tumor. Diagnosis is based on clinical evaluation, radiological findings, histopathological features in conjunction with immunostaining with specific neuroendocrine markers such as chromogranin, synaptophysin, CD56, and markers of epithelial differentiation such as cytokeratin, CDX2, and carcinoembryonic antigen. A 50 -year-old female presented with a history of dysphagia, chest pain, anorexia, and significant weight loss with normal physical findings and baseline investigations. Upper Gastrointestinal endoscopy showed growth at the gastroesophageal junction involving cardia of the stomach. Histopathological examination of the resected mass showed both adenocarcinoma and neuroendocrine carcinomatous components each involving more than $30 \%$ of the total mass examined. Identifying adenocarcinoma component admixed with a high-grade neuroendocrine component is significant as the prognosis and survival of patients differ from pure adenocarcinoma.
\end{abstract}

\section{Correspondence:}

\section{Dr. Deepshikha Gaire}

Resident, Department of Pathology

Tribhuvan University Teaching Hospital, Kathmandu, Nepal

ORCID ID: 0000-0002-2512-3344

Email: dipc62@gmail.com

Reveived : May 21 $1^{\text {st }} 2020$; Accepted : September $15^{\text {th }} 2020$

Citation : Gaire D, Maharjan D, Sharma N. Mixed Adenoneuroendocrine carcinoma of proximal stomach; a rare but sinister pathology. J Pathol Nep 2020;10:1772-5. DOI: 10.3126/jpn.v10i2.29050

Copyright: This is an open-access article distributed under the terms of the Creative Commons Attribution 4.0 International License, which permits unrestricted use, distribution, and reproduction in any medium, provided the original author and source are credited.

\section{INTRODUCTION}

Mixed neuroendocrine-non neuroendocrine neoplasm (MiNEN) is defined as a mixed epithelial neoplasm composed of both neuroendocrine and non-neuroendocrine components with variable proportions of each component. The neuroendocrine component can show morphological features including well to poorly differentiated neuroendocrine neoplasms and the non-neuroendocrine component can include different tumor types depending on the site of origin. Mixed exocrine-neuroendocrine neoplasms can be grouped into different prognostic categories according to the grade of malignancy of each component. 
In the 2019 WHO classification of tumors of the digestive tract, mixed exocrine-neuroendocrine carcinomas are defined as MiNEN which is further classified as mixed adenocarcinoma-NEC (MANEC) and mixed adenocarcinoma-NET depending on the grade of the neuroendocrine component. ${ }^{1}$ MiNENs have been described in several organs like the stomach, pancreas, gall bladder, uterine cervix, and caecum. ${ }^{2,3}$ To date, only seven cases have been reported in the caecum. ${ }^{4}$ Development of immunohistological methods has contributed greatly to its recognition in daily diagnostic practice.

\section{CASE REPORT}

A 50-year-old female presented to the TUTH with dysphagia for 7 months, more for solid food, and retrosternal pain. It was associated with anorexia, hemoptysis, and significant weight loss. Physical examination showed no abnormalities. Baseline blood investigations were within normal limits. Upper Gastrointestinal endoscopy showed a mass in the gastroesophageal junction (GEJ) with cardia involvement. Further gastrograffin swallow was done which showed irregular narrowing of the esophagus at the GEJ. A biopsy was taken and histopathological examination revealed moderately differentiated adenocarcinoma. The patient underwent a total D2 gastrectomy without oncological pretreatment.

Gross examination of the surgical specimen revealed a proliferative mass measuring $7 \times 7 \mathrm{~cm}$ which was very close to the proximal resection margin and $8 \mathrm{~cm}$ from the distal resection margin. Mass was located in the cardia of the stomach extending up to the GEJ (the epicenter of the tumor was $>2 \mathrm{~cm}$ from GEJ). On gross examination, the mass was extending up to the muscular layer of the gastric wall.

Histopathological examination of Hematoxylin and
Eosin stained slides showed a tumor with two types of morphological patterns. About $65 \%$ of mass examined showed tumor cells forming neoplastic glands, tubules, cords, and solid sheets arising from mucosa and infiltrating up to the subserosa of the gastric wall. These tumor cells were round to oval with vesicular nuclear chromatin, prominent nucleoli, and a moderate amount of eosinophilic cytoplasm. These tumor cells exhibited a moderate degree of pleomorphism with multiple foci of necrosis and hemorrhage. About 35\% of the mass was composed of tumor cells arranged in solid sheets, trabeculae, and cords. These tumor cells were large with large round to ovoid nuclei, coarsely granular to vesicular nuclear chromatin, and prominent nucleoli, a scant amount of cytoplasm (fig. 1A and B). Mitosis constituted 30/10 HPF in the mitotically active area. Perineural invasion was noted. However, the lymphovascular invasion was not identified. All margins were free of tumor and none of the lymph nodes showed metastases (0/27); TNM staging (CAP protocol 2017, AJCC 8th edition) was $\mathrm{pT} 3 \mathrm{pN} 0$.

With suspicion of MANEC, IHC was done. Tumor cells of neuroendocrine morphology were strongly positive for chromogranin (fig. 1C) with a ki67 index of 30\%. With these findings, the final diagnosis of MANEC was made. Then the patient received postoperative chemotherapy. The patient has been followed up for 6 months since the surgery, and the last CT scan did not reveal any signs of recurrence.

\section{DISCUSSION}

There is a wide spectrum of a combination of nonneuroendocrine and neuroendocrine components in the gastrointestinal tract, ranging from neuroendocrine neoplasms with a focal exocrine component at one extreme to exocrine carcinomas with interspersed neuroendocrine cells at the other. However, mixed non-neuroendocrine-
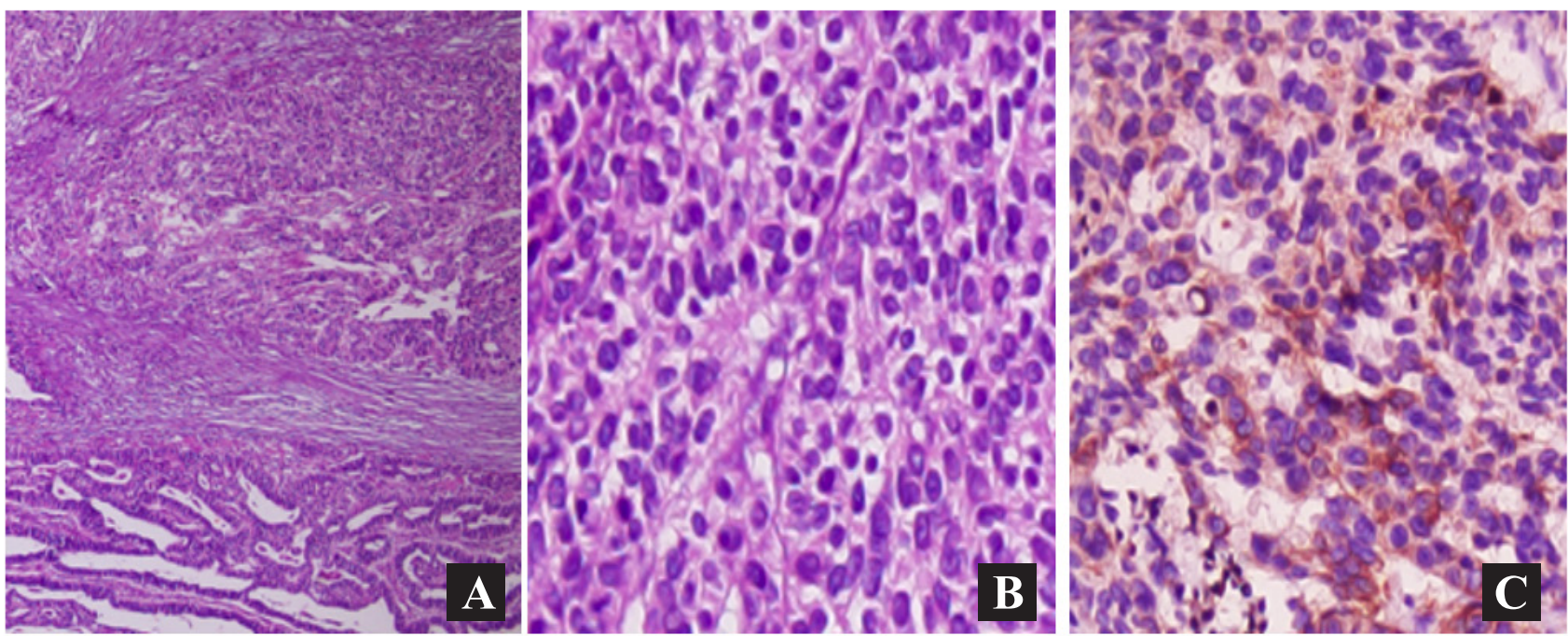

Figure 1A: Section showed adenocarcinoma component along with the neuroendocrine component (HE stain, X200). 1B: An area showing high-grade neuroendocrine carcinoma (HE stain, X400). 1C: Immunohistochemical stain showing tumor cells with neuroendocrine pattern was positive for chromogranin (Immunoperoxidase X400) 
neuroendocrine tumors are only those neoplasms in which each component represents at least 30\% percent of the lesion. In addition, both the non-neuroendocrine and the neuroendocrine components can have different morphological features, ranging from adenomas to adenocarcinomas with different degrees of differentiation in exocrine components and from well-differentiated to poorly differentiated neuroendocrine tumors in neuroendocrine components. ${ }^{5}$ In 2019 WHO classification of tumors of the digestive tract, mixed exocrine-neuroendocrine carcinomas are defined as MiNEN which is further classified as MANEC, and mixed adenocarcinoma-NET depending on the grade of the neuroendocrine component.

Unlike the index case, the mean age of presentation of MANEC is 60 years and males are more affected than females. ${ }^{6}$ Presenting symptoms of the patient with pure adenocarcinoma or pure neuroendocrine carcinoma are alike, so difficult to distinguish clinically. The bulk of cases with MANEC presents with non-specific symptoms leading to land up often in an advanced stage with distant metastasis. The index case was also presented with non-specific symptoms like dysphagia, anorexia, weight loss, chest pain, and hemoptysis. The majority of gastric MANECs show aggressive behavior and poor prognosis. The tumor behavior depends upon the most aggressive component, which is usually the neuroendocrine one. ${ }^{6}$

MANEC has been reported in various sites like the gall bladder, biliary tree, pancreas, esophagus, stomach, colon, and uterine cervix. About the distribution in the stomach equal distribution of the tumor in the upper and lower parts of the stomach was reported in one study. ${ }^{7}$ Macroscopically, these neoplasms appear solid, polypoid, nodular, or papillary, with a mean size of about $5 \mathrm{~cm}$. It appears grey-white on cut surfaces as accord with the index case who presented with large proliferative mass. Focal area of hemorrhage, necrosis, and mucin filled area may be evident on gross examination. Microscopically, MANEC comprises high-grade neuroendocrine neoplasm admixed with adenocarcinoma. Adenocarcinoma components can be tubular, papillary, mucinous, or other types of low to a high grade of differentiation. Neuroendocrine carcinoma is composed of large, poorly formed trabeculae or sheets of poorly differentiated cells. They can be subtyped as small cell NEC (SCNEC) or large cell NEC (LCNEC). LCNEC is composed of large cells with vesicular nuclei showing prominent nucleoli and abundant eosinophilic cytoplasm. SCNEC is composed of neoplastic cells with scant cytoplasm and hyperchromatic nuclei without nucleoli. NECs show a mitosis rate of $>20$ mitoses $/ \mathrm{mm} 2$ and ki67 index of $>20 \%$.

The latest developments in immunohistochemical staining techniques have led to an increased frequency of identifying few neuroendocrine cells in adenocarcinomas in the tumor tissues. Both small and large cell neuroendocrine components are positive for synaptophysin, chromogranin
A, or CD56. At least two out of three commonly used neuroendocrine markers must be abundantly expressed for diagnosis of MANEC. ${ }^{8}$ Diagnostic criteria of Ki67 or MIB1 for MANEC is $>20 \%$; however, the index is usually very high $(>60 \%)$. Our case was with high-grade NEC with large cell morphology with positive staining for chromogranin and ki67 index of 30\%. Due to the lack of any specific radiologic or symptomatic predictors of the tumor, the diagnosis is made based on the histopathological findings and immunohistochemical study.

MANECs are rare tumors diagnosed postoperatively using immunohistochemical evaluation. Although the presentation and surgical management are similar to that of pure adenocarcinomas or NECs, patients must undergo postoperative multidisciplinary oncologic and surgical management as soon as the tumor is diagnosed. After the diagnosis of MANEC was made in our patient, she was treated with postoperative chemotherapy. Since MANEC is notorious for metastasis and high recurrence, chemotherapy has been reported to be the treatment of choice. Chemotherapy has improved the median survival to a range of 6 to 12 months and occasional long-term survival. ${ }^{9}$ Likewise, a study done on MANEC in China showed worse survival and higher recurrence of MANECs. ${ }^{10}$ Thus early recognition of MANEC is a crucial step as they carry poor prognosis and survival.

\section{CONCLUSIONS}

Since MANEC is an aggressive pathology with a grave prognosis, all efforts should be given to diagnose it at the earliest using immunological techniques for prompt management.

\section{Conflict of Interest: None}

\section{REFERENCES}

1. La Rosa S, Rindi G, Solcia E, Tang LH. WHO classification of tumours of digestive system. 5th ed. IARC press: Lyon; 2019. pp 106.

2. Pham QD, Mori I, Osamura RY. A case report: gastric mixed neuroendocrine-nonneuroendocrine neoplasm with aggressive neuroendocrine component. Case Rep Pathol 2017;9871687. Crossref

3. Kwok CM. Mixed adenoneuroendocrine carcinoma of the stomach. Case Rep Gastroenterol 2015;9:241-5. Crossref

4. Gurzu S, Kadar Z, Bara T, et al. Mixed adenoneuroendocrine carcinoma of gastrointestinal tract: report of two cases. World J Gastroenterol 2015;21:1329-33. Crossref

5. Richards D, Davis D, Yan P, et al. Unusual case of small cell gastric carcinoma: Case report and literature review. Dig Dis Sci. 2011;56:951-7. Crossref

6. Scardoni M, Vittoria E, Volante M, et al. Mixed adenoneuroendocrine carcinomas of the gastrointestinal tract: targeted next generation sequencing suggests a monoclonal origin of the two components. Neuroendocrinology 2014;100:310-6. Crossref

7. La Rosa S, Inzani F, Vanoli A, et al. Histologic characterization 
and improved prognostic evaluation of 209 gastric neuroendocrine neoplasms. Hum Pathol. 2011;42:1373-84. $\underline{\text { Crossref }}$

8. Tanabe T, Nishimaki T, Kanda T, et al. Esophageal composite carcinoma with tripartite differentiation: clinicopathological analysis of three cases. Esophagus. 2005;2:91-6. Crossref

9. Richards D, Davis D, Yan P, et al. Unusual case of small cell gastric carcinoma: Case report and literature review. Dig Dis Sci. 2011;56:951-7. Crossref
10. Xie JW, Lu J, Wang JB, et al. Prognostic factors for survival after curative resection of gastric mixed adenoneuroendocrine carcinoma: a series of 80 patients. BMC cancer 2018;18:1021. Crossref 